\title{
Comparative Analysis of Color Image Watermarking Technique in RGB, YUV, and YCbCr Color Channels
}

\author{
Roshan Koju and Shashidhar Ram Joshi \\ Department of Electronics and Computer Engineering \\ Institute of Engineering, Tribhuwan University \\ e-mail: contactroshankoju@gmail.com
}

\begin{abstract}
Since there are a number of color spaces, it has always been a big question to choose one for watermarking. The aim of this work is to find out better color space, among the frequently used one, under the same condition. Comparative performance analysis of color image watermarking technique in color channels of RGB, YUV, YCbCr color spaces was studied. For this purpose, color channels were watermarked using single level discrete wavelet transform-singular value decomposition (DWT-SVD). PSNR, and SSIM were used to test the imperceptibility of watermarked images. PSNR and NCC were used to measure the similarity of extracted and original watermarks. The maximum recorded PSNR value is 62.372 for R channel of RGB color space with SSIM value equal to 0.9709 . Color channels of $\mathrm{YCbCr}$ color space were observed to be more robust and transparent as watermark image is best recovered from YCbCr color space with NCC values in the range 0.86 to 0.877 and SSIM values in the range 0.546 to 0.554 under various geometric attacks.
\end{abstract}

Key words: Cover image, digital watermark, DWT-SVD, imperceptibility, robustness

\section{Introduction}

With rapid development in internet and multimedia technologies, electronic distribution of image, audio, video and data have become easier. This reality, however, has at the same time led to increased concern about the protection of the intellectual property rights of the content that is distributed in electronic form. Since electronic data can be stored and transmitted efficiently, effectively and economically, they can be copied without any loss of fidelity that exactly resembles the original one. They can also be edited easily as one can access the exact discrete locations that need to be changed (Saxena 2008).

Digital image watermarking is one of the techniques for solving copyright and ownership issues. Digital watermark is a pattern of bits inserted into a digital image, audio, video or text file that identifies the file's copyright information (Low and Lapone 1998). Robustness, imperceptibility, complexity and blind watermarking are four essential factors to determine quality of watermarking scheme (Ming-Shing et al 2001). Most of the researches have been conducted in binary or gray images. Comparatively, less watermarking algorithms are found for color images (Xiaohui et al 2011). In addition, when it is a matter of color image, there is always freedom to choose different color channels like R,G,B or Y,U,V or $\mathrm{Y}, \mathrm{Cb}, \mathrm{Cr}$ or Y,I,Q or C,M,Y,K to embed watermarks.

Some of researches have used RGB (red, green and blue) color space for embedding watermarks. RGB is used in computer graphic applications. Nonlinear RGB is also used in image processing, JPEG, MPEG standards. First, R, G, B planes are separated and either one of these planes or combination of two can be used for embedding. But, RGB color space is complex in describing the color pattern and has redundant information between each component. Since pixel values in RGB color space are highly correlated, RGB color space is converted into other color spaces (Sulong et al 2012). 
The YUV color model defines a color space in terms of one luma (Y) and two chrominance (UV) components. YUV color space is converted from RGB color space using the following equations:

$$
\begin{aligned}
& Y=0.299 R+0.587 G+0.114 B \\
& U=-0.147 R-0.289 G+0.436 B \\
& V=0.615 R-0.515 G-100 B
\end{aligned}
$$

Similarly YUV is converted back into RGB using following equations:

$$
\begin{aligned}
& R=Y+1.140 \mathrm{~V} \\
& G=Y-0.395 U-0.581 \mathrm{~V} \\
& B=Y+2.032 \mathrm{~V}
\end{aligned}
$$

$\mathrm{YCbCr}$ is another color model in which $\mathrm{Y}$ denotes the luminance component. It means that $\mathrm{Y}$ shows the brightness (luma). Also both of $\mathrm{Cb}$ and $\mathrm{Cr}$ represent the chrominance factors. This model is considered as scaled and offset version of YUV. It is different from YUV in the sense that YUV is analog system while $\mathrm{YCbCr}$ is digital system. This color model is consistent to human visual system (HVS) (Fengmei Liang 2011). YUV and YCbCr color spaces were originally developed for color TV broadcasting. These color spaces are used in image and video compression applications such as MPEG-1, MPEG-2, MPEG-4, H.261, H.262, H.263, H.264, JPEG, JPEG-2000 etc.

$\mathrm{RGB}$ to $\mathrm{YCbCr}$ conversion can be obtained from the following equations

$$
\begin{aligned}
& Y=0.299 R+0.587 G+0.114 B \\
& C b=0.596 R-0.272 G-0.321 B \\
& C r=0.212 R-0.523 G-0.311 B
\end{aligned}
$$

Similarly, YCbCr can be converted back into RGB from following equations:

$$
\begin{aligned}
& R=Y+0.956 C b+0.620 C r \\
& G=Y-0.272 C b-0.647 C r \\
& B=Y-1.108 C b+1.705 C r
\end{aligned}
$$

As these color formats are developed for different purposes, definitely embedding watermark in these color channels may not result the same performance.
Thus, this paper compared the performance of three commonly used color spaces RGB, YUV and YCbCr. Watermark was embedded via discrete wavelet transform- singular value decomposition (DWT-SVD) scheme.

Discrete wavelet transform decomposes an input image into four non-overlapping multi-resolution coefficient sets, a lower resolution approximation image (LL) as well as horizontal (HL), vertical (LH) and diagonal (HH) detail components. The sub-band LL represents the coarse-scale DWT coefficients while the coefficient sets LH, HL and HH represent the finescale of DWT coefficients. To obtain the next coarser scale of wavelet coefficients, the sub-band LL is further processed until some final scale $\mathrm{N}$ is reached. This process is shown in Fig. 1.

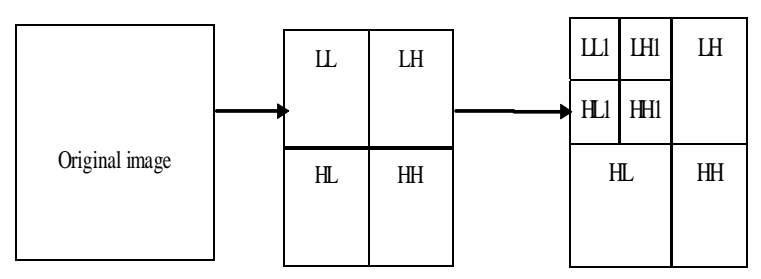

Fig. 1. DWT Decomposition of image

Due to its excellent spatio-frequency localization properties, the DWT is very suitable to identify the areas in the host image where a watermark can be embedded effectively (Guo and Georganas 2002).

In general most of the image energy is concentrated at LL sub bands and therefore embedding watermarks in these coefficient sets may degrade the image significantly. Embedding in the low frequency coefficient sets, however, could increase robustness significantly. On the other hand, HH sub bands include the edges and textures of the image and the human eye is not generally sensitive to changes in such coefficient sets. This allows the watermark to be embedded without being perceived by the human eye. (Guo and Georganas 2002).

Singular values of the image gives very good stability. When a small value is added, it does not result too much variation. Hence Singular Value decomposition in linear algebra is used to solve many mathematical problems (Yin 2007). 
Every real matrix A can be decomposed into product of three matrices $A=U^{\prime \prime} V^{T}$, where $U$ and $V$ are orthogonal matrices such that, $\mathrm{UU}^{\mathrm{T}}=1$ and $\mathrm{VV}^{\mathrm{T}}=$ 1 and " is summation of diagonal entries $\lambda 1, \lambda 2 \cdots$ gives the singular vectors of $\mathrm{A}$. These diagonal entries are called as Singular Values of A and the decomposition is called as 'Singular Value Decomposition'. Singular Values specifies the luminance of an image layer while corresponding pair of singular vectors specifies the geometry of the image layer. (Yin, 2007). The rest of the paper is organized as follows: section II focuses on methodology that discusses the common platform of testing mechanism. Section III discusses about result and discussion of the research work.

\section{Methodology}

Different authors have implemented different color channels for watermarking. But there must be a mechanism to test these color channels in the same platform to determine which one performs better. For this process an environment was set in which same watermark image was embedded in the same fashion in all color spaces. Before embedding watermark image, the color components of RGB, YCbCr and YUV color space were separated. Then each color channel was processed to embed and extract watermark image as follows:

A) Watermark embedding algorithm

i. Read color cover image (convert it into YUV or $\mathrm{YCbCr}$ color space if required)

ii. Separate each color channels from each color space

iii. Select one of color channel and apply 1-level DWT

iv. Select LL sub-band and apply SVD to that sub band resulting U,S,V

v. Read grey watermark image and apply 1level DWT

vi. Select LL sub band and apply SVD to that sub band resulting $\mathrm{U}_{\mathrm{W}} \mathrm{S}_{\mathrm{W}} \mathrm{V}_{\mathrm{W}}$

vii. Add watermark to cover image using following relation

$\mathrm{S}_{\mathrm{NEW}}=\mathrm{S}+k * \mathrm{~S}_{\mathrm{W}}$ where $k$ is flexing factor

viii. Apply reverse SVD to form new LL subband with new singular values

ix. Apply inverse DWT to obtain watermarked color channel

x. Combine three color channels to form watermarked image
B) Watermark extraction algorithm

i. Read color watermarked image and separate its color channels

ii. Select respective color channel used in embedding and apply 1-DWT transform

iii. Select LL sub band and apply SVD to get

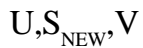

iv. Read watermark image and apply 1-DWT and select LL sub band for applying SVD that results $\mathrm{U}_{\mathrm{W}} \mathrm{S}_{\mathrm{W}} \mathrm{V}_{\mathrm{W}}$

v. Extract watermark using following relation

vi. $\mathrm{S}_{\text {EXTRACT }}=\left(\mathrm{S}_{\text {NEW }}-\mathrm{S}_{\mathrm{W}}\right) / k$

vii. Form Extracted watermark image with this new singular values.

\section{Results and Discussion}

Above discussed algorithms were implemented in Matlab and analyzed with all color channels of RGB, YUV and YCbCr. Common platform, 1-level DWTSVD, was used to embed watermark in cover image. Two major tests, imperceptibility and robustness against attacks, were conducted with all color channels. Imperceptibility is measure of transparency and was measured via statistical test like peak signal to noise ratio (PSNR) and structural similarity (SSIM).

PSNR takes signal strength into consideration in addition to error. Mathematically, PSNR is given by following relation:

$P S N R=10 \log _{10\left(\mathrm{MAX}^{2}\right.}^{2}$ MSE)

Where, MSE is mean square error.

Higher is the PSNR value, better is the image quality. When PSNR is higher than $35 \mathrm{~dB}$ watermarked image has a very good quality and the eye could hardly tell the difference between the original and the watermarked image.

PSNR is totally statistical and has nothing to do with HVS. SSIM is one of the measures that tries to address this problem of PSNR (Z.Wang, 2004). SSIM is mathematically expressed as:

$S S I M=\frac{(2 \mu x \mu y+c 1)(2 \sigma x y+c 2)}{\left(\mu x^{\wedge} 2+\mu y^{\wedge} 2+c 1\right)\left(\sigma x^{\wedge} 2+\sigma y^{\wedge} 2+c 2\right)}$ (6) 
Where $\mu, \sigma, \sigma_{\mathrm{xy}}$ are mean, variance and covariance of images and $\mathrm{c} 1$ and $\mathrm{c} 2$ are stabilizing constants. SSIM has value ranges in the 0 to 1 . Highly similar images will have SSIM value near to 1 .

Robustness of image was tested by comparing similarity of extracted watermark image with original watermark image. Similarity of watermarks were carried out on the basis of normalized cross correlation (NCC) and SSIM. Mathematical relation for NCC is expressed as follows:

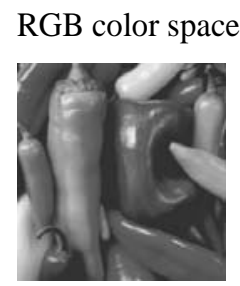

Original image

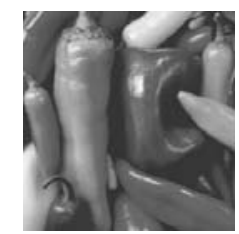

$\mathrm{R}$

channel

Watermarked

\begin{abstract}
Watermarked
\end{abstract}
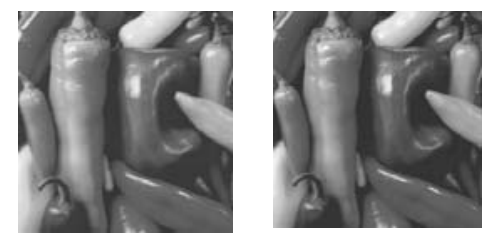

Where $\mathrm{W}$ was original mark and $\mathrm{W}$ ' was extracted mark, M, N were rows and column of watermark image. Fig. 2 below shows the original and watermarked images. PSNR and SSIM values of watermarked image and original cover image is shown in Table 1.

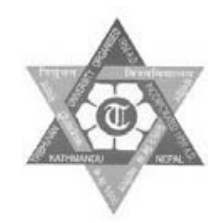

channel Watermark

Image

YUV color space

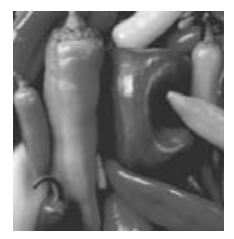

Original image

YCbCr color space

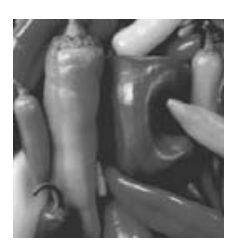

Original image

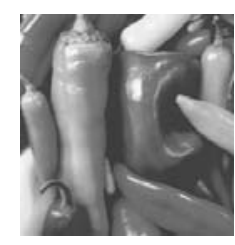

Y

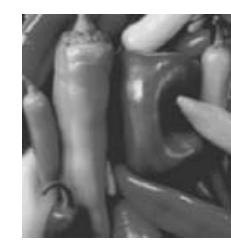

channel U channel V

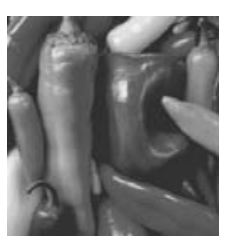

channel Watermark

\section{Watermarked}

Watermarked

Watermarked

Image

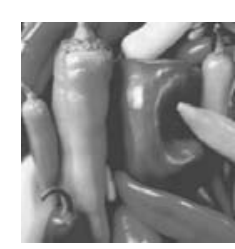

Y channel

Watermarked

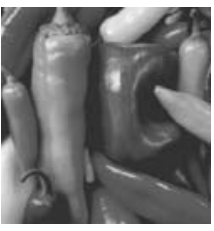

$\mathrm{Cb}$ channel $\mathrm{Cr}$

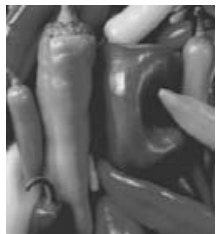

channel Watermark

Watermarked Image

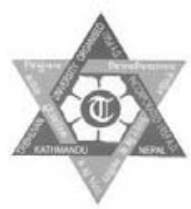

Watermarked

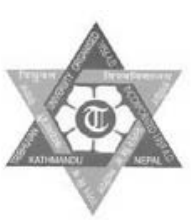

Fig. 2. Original cover image and watermarked images

Table1. Imperceptibility test

\begin{tabular}{l|l|l|l|l|l|l|l|l|l}
\hline Parameters & R & G & B & Y & U & V & Y & Cb & Cr \\
\hline PSNR & 62.372 & 60.0397 & 59.796 & 49.4368 & 53.1081 & 60.5676 & 46.4279 & 45.0831 & 49.9651 \\
SSIM & 0.9709 & 0.9641 & 0.9811 & 0.8978 & 0.9595 & 0.9544 & 0.9423 & 0.9998 & 0.98 \\
\hline
\end{tabular}


Roshan Koju and Shashidhar Ram Joshi/Comparative Analysis.......

The maximum recorded value of PSNR was 62.372, in $\mathrm{R}$ channel. However, all watermarked images had PSNR value greater than $35 \mathrm{~dB}$. This shows all channels of defined color spaces result transparent watermarking. Similar result was reflected in SSIM values. It was near to 1 for all color channels indicating watermarked images were highly transparent irrespective to color channels.

The watermarks extracted after various attacks are shown in following Fig.3. The NCC and SSIM values of extracted and original watermarks are presented in Table 2 and 3.

\begin{tabular}{|c|c|c|c|c|c|}
\hline & Grayscale & Rotate & $\begin{array}{l}\text { Salt and pepper } \\
\text { (SP) noise }\end{array}$ & Contrast & Crop \\
\hline R channel watermerked & ${ }^{a}$ & t. & & & \\
\hline G channel watermerked & a & & & & \\
\hline B channel watermerked & + & & & & 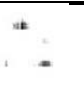 \\
\hline Y channel watermerked & & & & & \\
\hline U channel watermerked & $\dot{s}$ & & & & \\
\hline V channel watermerked & $\stackrel{1}{0}$ & & & & \\
\hline Y channel watermerked & & & & & \\
\hline $\mathrm{Cb}$ channel watermerked & & & & & \\
\hline $\mathrm{Cr}$ channel watermerked & & & & & $\frac{1}{9}$ \\
\hline
\end{tabular}

Fig. 3. Extracted watermarks after various attacks on watermarked image

Table 2. NCC values of extracted and original watermark

\begin{tabular}{l|l|l|l|l|l|l|l|l|l}
\hline Attacks & $\mathrm{R}$ & $\mathrm{G}$ & $\mathrm{B}$ & $\mathrm{Y}$ & $\mathrm{U}$ & $\mathrm{V}$ & $\mathrm{Y}$ & $\mathrm{Cb}$ & $\mathrm{Cr}$ \\
\hline Gray & 0.321 & 0.291 & 0.334 & 0.263 & 0.334 & 0.357 & 0.864 & 0.877 & 0.875 \\
SP Noise & 0.179 & 0.251 & 0.763 & 0.253 & 0.001 & 0.301 & 0.864 & 0.861 & 0.864 \\
Rotate & 0.176 & 0.251 & 0.975 & 0.252 & 0.001 & 0.285 & 0.864 & 0.860 & 0.861 \\
Crop & 0.169 & 0.222 & 0.306 & 0.218 & 0.001 & 0.303 & 0.861 & 0.860 & 0.860 \\
Contrast & 0.121 & 0.121 & 0.164 & 0.119 & 0.001 & 0.366 & 0.860 & 0.864 & 0.861 \\
\hline
\end{tabular}


Nepal Journal of Science and Technology Vol. 15, No.2 (2014) 133-140

Table 3. SSIM values of extracted and original watermark

\begin{tabular}{l|l|l|l|l|l|l|l|l|l}
\hline Attacks & $\mathrm{R}$ & $\mathrm{G}$ & $\mathrm{B}$ & $\mathrm{Y}$ & $\mathrm{U}$ & $\mathrm{V}$ & $\mathrm{Y}$ & $\mathrm{Cb}$ & $\mathrm{Cr}$ \\
\hline Gray & 0.224 & 0.219 & 0.224 & 0.204 & 0.213 & 0.24 & 0.554 & 0.548 & 0.546 \\
SP Noise & 0.032 & 0.052 & 0.092 & 0.074 & 0.106 & 0.051 & 0.554 & 0.554 & 0.554 \\
Rotate & 0.07 & 0.168 & 0.732 & 0.169 & 0.106 & 0.075 & 0.554 & 0.554 & 0.554 \\
Crop & 0.062 & 0.12 & 0.105 & 0.116 & 0.106 & 0.039 & 0.552 & 0.554 & 0.554 \\
Contrast & 0.06 & 0.045 & 0.058 & 0.03 & 0.106 & 0.642 & 0.554 & 0.554 & 0.554 \\
\hline
\end{tabular}

NCC values of extracted watermark and original watermark were in the range from 0.86 to 0.877 for $\mathrm{YCbCr}$ color space while that for YUV were in the range 0.001 to 0.366 and for $\mathrm{RGB}$, they were in the range 0.121 to 0.334 . Similarly, SSIM values were in the range from 0.546 to 0.554 which was much greater than that of other color spaces.

It indicates, embedding watermark in $\mathrm{YCbCr}$ color space is more robust and imperceptible in comparison to rest of two. Gao et al (2013), and Yang (2009) have embedded digital watermark using RGB color scheme. They chose B and $\mathrm{G}$ channels to embed the watermark. They only used 2-DWT and watermarked image had good transparency (almost 1). The extracted watermark was almost the same as original watermark which might be due to Arnold transform or discrete cosine transform of watermark that they had adopted before embedding. Similarly, (Benhocine and Laouamer 2013) used RGB color space where cover image and watermark image were passed via SVD and embedded using linear interpolation. The method was highly imperceptible (with almost $1 \mathrm{NC}$ ) but not so robust against the attacks which might be due to application of SVD only.

Y channel of $\mathrm{YCbCr}$ color space was used to embed watermark in (Yesilyurt et al 2013) using middle band coefficients exchange method. The method has lower imperceptibility PSNR value $40.18 \mathrm{~dB}$ and robust to various geometric attacks and Jpeg compression. Similarly, (Khalili 2011) used wavelet-based watermarking approach for hiding watermark image in color host images was proposed. In this work Y channel of $\mathrm{YCbCr}$ color space was used to embed watermark using code division multiple access (CDMA) scheme which was found to be imperceptible (with correlation $=0.9992$ ) and robust against Jpeg compression and noise attacks.
Gunjal (2011) used Y channel of YUV color scheme to watermark using DWT transform. Pseudorandom (PN) sequence was also used to scramble watermark image before embedding into host image. The scheme resulted in exact recovery of watermark with standard database images of size $512 \times 512$, giving correlation factor equal to 1 and robust against some of geometric attacks. Similarly, Bkhetan and Awa (2013) chose V channel of YUV color space to embed watermark after passing cover image through DWT transform. The discussed method was found to be resistant against few geometric attacks like noise and filters. In another work (Ming-Shing and Tseng 2006), Y channel of YUV was used to embed watermark along with adaptive entropy casting and DWT. Here transparency observed was NC $=0.9852$. Similarly, this method had higher robustness. This higher value might be due to different mechanisms of embedding watermark.

In this paper, performance of watermarking in different color channels of RGB, YUV and $\mathrm{YCbCr}$ were compared under common platform. It was observed that embedding watermarks in color channels of $\mathrm{YCbCr}$ were more robust and imperceptible than other color channels.

\section{Acknowledgements}

I would like to thank fresh graduate $\mathrm{PhD}$ fellowship program, University Grant Commission for providing the research grant. We are also thankful to Research Department, Institute of Engineering, Pulchowk, Tribhuwan University for support and guidance.

\section{References}

Benhocine, A. and L. Laouamer. 2013. New watermarking scheme based on singular value decomposition. Journal of Information Hiding and Multimedia Signal Processing, 4(1) : 9-18. 
Roshan Koju and Shashidhar Ram Joshi/Comparative Analysis........

Bkhetan, Z. A. and N. Awa 2013. Robust color image watermarking and web tracing system using digital wavelet transform and mobile agents. International Arab Journal of e-Technology, 3(2):99-106

Gao, H. and N. Georganas 2002. Multi-resolution image watermarking scheme in the spectrum domain. In: Proceedings of IEEE Canadian Conference on Electrical and Computer Engineering, (May) Canada:873-878.

Gao H. and L. Jia and M.Liu 2013. A digital watermarking algorithm for color image. Telkomnika, 11(6) : $3271-3278$

Gunjal, B. L. 2011. Wavelet based color image watermarking scheme giving high robustness and exact correlation. International Journal of Emerging Trends in Engineering and Technology, 1(1) : 21-30

Khalili, M. 2011. A novel secure, impercptible and robust cdma digital image watermarking in jpeg-YCbCr channel using DWT2. International Journal of Enterprise Computing and Business system, 1(2).

Liang, Fengmei, and L. Wang 2011. An improved waveletbased color image watermark algorithm . Journal of Computational Information Systems, 6(7) :20132020.

Low, S.H and A.M. Lapone 1998. Document identification for copyright protection using centroid detection. IEEE Trans. on Communications, 46(3) :372-383.

Ming-Shing, H., D.C. Tseng and Y.H Huang. 2001. Hiding Digital watermarks using multi resolution wavelet transform. IEEE Transactions on Industria, 48(5) : 875-882
Ming-Shing, H. and D.C. Tseng 2006. Wavelet-based color image watermarking using adaptive entropy casting. In: Proceedings of IEEE International Conference on Multimedia and Expo (July 9-12, 2006), Toronto, Canada, 1593-1596.

Saxena, V. 2008. Digital image watermarking. $\mathrm{PhD}$ thesis ,Jaypee institute of information and technology, India.

Sulong, G. B, H. Hasan, A. Selamat, M. Ibrahim and Saparudin 2012. A new color image watermarking technique using hybrid domain. International Journal of Computer Science Issues, 9(6) :109-114.

Wang Z, A. Bovik,H.R. Seikh and E.P. Simoncelli 2004. Image quality assessment: from error visibility to structural similarity. IEEE Transactions of Image Processing 13(4):1-12.

Xiaohui M., Y. Hanmin and H.Tan. 2011. The application of hybrid encryption and HVS in the digital . Software Newsletter, 10(2):57-59.

Yang W.M and Z. Jin. 2009. A watermarking algorithm based on wavelet and cosine transform for color Image. First International Workshop on Education Technology and Computer Science.2:899-903

Yesilyurt M., Y. Yalman and A.T. Ozcent 2013. A DCT based watermarking method using luminance component. Elektronika ir electrotechnika, 19(4) : 47-52.

Yin, Chen qun, and A. Li Li, 2007. Color image watermarking algorithm based on DWT-SVD. In: Proceedings of the IEEE international conference on automation and logistics (August 1821,2007)Jinan, China: 2607-2611. 
Nepal Journal of Science and Technology Vol. 15, No.2 (2014) 133-140 